\title{
IMPLEMENTATION OF THE GMRES METHOD USING HOUSEHOLDER TRANSFORMATIONS*
}

\author{
HOMER F. WALKER $\dagger$
}

\begin{abstract}
The standard implementation of the GMRES method for solving large nonsymmetric linear systems involves a Gram-Schmidt process which is a potential source of significant numerical error. An alternative implementation is outlined here in which orthogonalization by Householder transformations replaces the Gram-Schmidt process. This implementation requires slightly less storage but somewhat more arithmetic than the standard one; however, numerical experiments suggest that it is more stable, especially as the limits of residual reduction are reached. The extra arithmetic required may be less significant when products of the coefficient matrix with vectors are expensive or on vector and, in particular, parallel machines.
\end{abstract}

Key words. GMRES method, iterative methods, matrix-free methods, nonsymmetric linear systems, Householder transformations.

AMS(MOS) subject classifications. $65 \mathrm{~F} 10,65 \mathrm{~N} 20$

1. Introduction. Of interest here is the generalized minimal residual (GMRES) method of Saad and Schultz [8]. This is an iterative method for solving large linear systems of equations

$$
A x=b
$$

in which $A \in \mathbf{R}^{n \times n}$ is nonsymmetric. For a full description of this method and its standard implementation, see [8]. For examples of its successful application to problems arising from the numerical solution of ordinary and partial differential equations, see Brown and Hindmarsh [2] and Wigton, Yu and Young [10].

In brief, the GMRES method begins with an initial approximate solution $x_{0}$ and initial residual $r_{0}=b-A x_{0}$; at the $m$ th iteration, a correction $z_{m}$ is determined in the Krylov subspace

$$
\mathcal{K}_{m}(v) \equiv \operatorname{span}\left\{v, A v, \ldots, A^{m-1} v\right\}
$$

with $v=r_{0}$ which solves the least-squares problem

$$
\min _{z \in \mathcal{K}_{m}\left(r_{0}\right)}\left\|b-A\left(x_{0}+z\right)\right\|_{2} .
$$

The $m$ th iterate is then $x_{m}=x_{0}+z_{m}$. The correction $z_{m}$ is chosen to reduce the residual norm as much as possible among the space of allowable corrections, and it is clear that the residual norm is nonincreasing from one iteration to the next. Furthermore, one can show that if exact arithmetic were used, then the solution would be reached in no more than $n$ iterations [8, Corollary 3, p. 865].

* Received by the editors December 22, 1986; accepted for publication (in revised form) May 3, 1987. This research was supported in part by the United States Department of Energy under contract DE-FG02-86ER25018 with Utah State University and in part by the Computing and Mathematics Research Division, Lawrence Livermore National Laboratory, Livermore, California 94550.

$\dagger$ Department of Mathematics, Utah State University, Logan, Utah 84322-3900. 
A natural approach to determining $z_{m}$ is to determine a basis of $\mathcal{K}_{m}\left(r_{0}\right)$ and then to solve an $m$-dimensional least-squares problem for the coefficients of the linear combination of basis elements that solves (1.2). Since the method is likely to proceed for a number of iterations, a basis should be determined for each $m$ in a way which allows economizing on the arithmetic incurred in incrementing $m$, e. g., by allowing updating of the matrix factorizations used to solve the least-squares problems for increasing values of $m$. An obvious choice of a basis for each $m$ is $\left\{r_{0}, A r_{0}, \ldots, A^{m-1} r_{0}\right\}$, and the solution of the least-squares problems for these bases can be carried out economically as $m$ is incremented (see Walker [9]). However, in many applications these least-squares problems are likely to be very ill-conditioned even for fairly small values of $m$, with a resulting degradation of the performance of the method. The GMRES implementation of [9], which uses these bases in conjunction with very stable Householder transformations, was observed by Hindmarsh and Walker [5] to perform significantly worse in stiff ODE solving experiments than the standard implementation of [8]. We also found this implementation to perform far worse on the two numerical problems outlined below than either the standard implementation or the implementation using Householder transformations given in the sequel, although details of the performance of this implementation are not shown here. Consequently, the use of the bases $\left\{r_{0}, A r_{0}, \ldots, A^{m-1} r_{0}\right\}$ is not recommended.

The implementation of GMRES given by Saad and Schultz [8] determines $z_{m}$ by maintaining for each $m$ a particular orthonormal basis $\left\{v_{1}, \ldots, v_{m}\right\}$ of $\mathcal{K}_{m}\left(r_{0}\right)$ together with an upper-Hessenberg $(m+1) \times m$ matrix $H_{m}$. These are generated through Arnoldi's method, the basic form of which is the following:

ARNOLDI'S METHOD. Suppose $v_{1}$ is given with $\left\|v_{1}\right\|_{2}=1$.

For $m=1,2, \ldots$, do:

a. Set

$$
\begin{aligned}
& h_{i m}=\left(A v_{m}\right)^{T} v_{i}, i=1,2, \ldots, m, \\
& \hat{v}_{m+1}=A v_{m}-\sum_{i=1}^{m} h_{i m} v_{i}, \\
& h_{m+1, m}=\left\|\hat{v}_{m+1}\right\|_{2},
\end{aligned}
$$

b. If $h_{m+1, m}=0$, then stop; otherwise, set

$$
v_{m+1}=\hat{v}_{m+1} / h_{m+1, m} \text {. }
$$

Clearly $\left\{v_{1}, \ldots, v_{m}\right\}$ generated by Arnoldi's method is an orthonormal basis of $\mathcal{K}_{m}\left(v_{1}\right)$ for each $m$. In the GMRES implementation of [8], $v_{1}=r_{0} /\left\|r_{0}\right\|_{2}$ and the $h_{i j}$ 's constitute the (possibly) nonzero elements of the matrices $H_{m}$. Note that

$$
A\left(v_{1}, \ldots, v_{m}\right)=\left(v_{1}, \ldots, v_{m+1}\right) H_{m}
$$

for each $m$.

With these orthonormal bases and upper-Hessenberg matrices, $z_{m}$ is determined as follows: Setting $z=\left(v_{1}, \ldots, v_{m}\right) y$ for $y \in \mathbf{R}^{m}$ gives

$$
\begin{aligned}
\min _{z \in \mathcal{K}_{m}\left(r_{0}\right)}\left\|b-A\left(x_{0}+z\right)\right\|_{2} & =\min _{y \in \mathbf{R}^{m}}\left\|r_{0}-A\left(v_{1}, \ldots, v_{m}\right) y\right\|_{2} \\
& =\min _{y \in \mathbf{R}^{m}}\left\|r_{0}-\left(v_{1}, \ldots, v_{m+1}\right) H_{m} y\right\|_{2} .
\end{aligned}
$$

Since $v_{1}$ is $r_{0} /\left\|r_{0}\right\|_{2}$ and since $\left\{v_{1}, \ldots, v_{m+1}\right\}$ is orthonormal, one has

$$
\min _{y \in \mathbf{R}^{m}}\left\|r_{0}-\left(v_{1}, \ldots, v_{m+1}\right) H_{m} y\right\|_{2}=\min _{y \in \mathbf{R}^{m}}\|\| r_{0}\left\|_{2} e_{1}-H_{m} y\right\|_{2}
$$


where $e_{1}$ is the first canonical basis vector in $\mathbf{R}^{m+1}$. It follows from (1.3) and (1.4) that $z_{m}=\left(v_{1}, \ldots, v_{m}\right) y_{m}$, where $y_{m}$ solves

$$
\min _{y \in \mathbf{R}^{m}}\|\| r_{0}\left\|_{2} e_{1}-H_{m} y\right\|_{2}
$$

In fact, this characterization of $z_{m}$ is valid even when $h_{m+1, m}=0$, which occurs if and only if the solution $y_{m}$ of (1.5) yields zero residual. Thus the GMRES method is forced to stop at the $m$ th iteration if and only if $x_{m}$ is the solution of (1.1).

Since $H_{m}$ is upper-Hessenberg, (1.5) is easily solved. In actual practice, $H_{m}$ is maintained in factored form, and the factors are updated with each increment of $m$. Typically, $H_{m}$ is factored as $H_{m}=Q_{m} R_{m}$, where $Q_{m}$ is a product of Givens rotations and $R_{m}$ is upper-triangular. In this case (1.5) is equivalent to

$$
\min _{y \in \mathbf{R}^{m}}\|\| r_{0}\left\|_{2} Q_{m}^{T} e_{1}-R_{m} y\right\|_{2}
$$

and $y_{m}$ is obtained by solving an upper-triangular system. Note that the absolute value of the last coordinate of $\left\|r_{0}\right\|_{2} Q_{m}^{T} e_{1}$ is just $\left\|b-A x_{m}\right\|_{2}$, and so the residual at the $m$ th iteration can be determined without actually having to compute the correction.

At the heart of Arnoldi's method is a Gram-Schmidt process, and so we refer to the GMRES implementation of [8] as the Gram-Schmidt implementation. The basic form of Arnoldi's method given above employs the classical Gram-Schmidt process, which is numerically untrustworthy. Because of roundoff, there may be severe loss of orthogonality among the computed $v_{m}$ 's. In practice, it is usual to implement Arnoldi's method using the modified Gram-Schmidt process (see Golub and Van Loan [4]). Mathematically, this is just a rearrangement of the classical process; computationally, it has superior properties.

Even the modified Gram-Schmidt process can fail to perform well if the vectors on which it operates are not sufficiently independent. Indeed, if $S=\left(s_{1}, \ldots, s_{m}\right)$ is an $n \times m$ matrix the columns of which are to be orthonormalized and if $Q=\left(q_{1}, \ldots, q_{m}\right)$ is the computed result of applying modified Gram-Schmidt to the columns of $S$ using floating point arithmetic with unit rounding error $\mathbf{u}$, then Bjorck [1] has shown that

$$
Q^{T} Q=I+E, \quad\|E\|_{2} \approx \mathbf{u} \kappa_{2}(S)
$$

where the condition number $\kappa_{2}(S)$ is the ratio of the largest singular value of $S$ to the smallest. It follows that at the $m$ th step of Arnoldi's method using modified GramSchmidt, $v_{m+1}$ may have a significantly nonzero component in the span of $\left\{v_{1}, \ldots, v_{m}\right\}$ if $\kappa_{2}\left(\left(v_{1}, \ldots, v_{m}, A v_{m}\right)\right)$ is large, i. e., if $A v_{m}$ is nearly in the span of $\left\{v_{1}, \ldots, v_{m}\right\}$. Saad [7, p. 214] has suggested that the Gram-Schmidt process in Arnoldi's method may be an important source of errors in the full and incomplete orthogonalization methods $([6],[7])$, which are related to GMRES.

There is an alternative orthogonalization procedure based on the use of Householder transformations which is reliable even if the vectors to be orthonormalized are not very independent. A Householder transformation is of the form $P=I-2 u u^{T}$, where $I$ is the identity matrix and $\|u\|_{2}=1$. We refer to $u$ as the Householder vector which determines $P$. Note that $P^{-1}=P^{T}=P$. Also, note that the action of $P$ on a vector or matrix can be easily determined using $u$; in particular, one need not explicitly form or store $P$ in the applications of interest here. If two vectors of the same norm are given, then it is easy to determine $P$ so that it takes one vector into the other; 
furthermore, if the first $k$ components of the two vectors are the same, then the $u$ which determines $P$ can be chosen so that its first $k$ components are zero. For more on the properties and uses of Householder transformations, see Golub and Van Loan [4].

To orthonormalize the columns of $S=\left(s_{1}, \ldots, s_{m}\right)$, one determines Householder transformations $P_{1}, \ldots, P_{m}$ such that $P_{m} \ldots P_{1} S=R$, an upper-triangular matrix. It is natural to determine $P_{1}, \ldots, P_{m}$ inductively by the requirement that $P_{k} \ldots P_{1}\left(s_{1}, \ldots, s_{k}\right)$ be upper-triangular for $k=1, \ldots, m$; if $P_{m} \ldots P_{1} S=R$, then this requirement is met if and only if for $k=2, \ldots, m$ the first $k-1$ components of the Householder vector determining $P_{k}$ are zero. Since $S=P_{1} \ldots P_{m} R$, the matrix $Q$ consisting of the first $m$ columns of $P_{1} \ldots P_{m}$ gives the desired orthonormalization of the columns of $S$. If $Q$ is computed in floating point arithmetic with unit rounding error $\mathbf{u}$, then (Bjorck [1])

$$
Q^{T} Q=I+E, \quad\|E\|_{2} \approx \mathbf{u}
$$

In view of the greater reliability of orthogonalization based on Householder transformations, as reflected in (1.6) and (1.7), it seems worthwhile to consider implementations of the GMRES method in which this orthogonalization replaces the GramSchmidt process in the implementation of [8]. We outline one such Householder implementation here. It is based on a particular method of using Householder transformations to generate orthonormal bases of Krylov subspaces. This method is essentially that used by Golub, Underwood and Wilkinson [3] in their implementation of the Lanczos algorithm; see also Golub and Van Loan [4, pp. 334-335]. An analogous implementation can be formulated without difficulty for the full orthogonalization method of Saad [6], but we do not give this here.

Our experiments indicate that the Householder implementation given here has better numerical properties than the Gram-Schmidt implementation, especially in the final iterations when the limits of residual reduction are neared. This Householder implementation uses slightly less storage than the Gram-Schmidt implementation; however, it requires additional arithmetic. The increase in arithmetic is always less than a factor of three, and we mention several important circumstances in which it may not be an overriding concern. We also give a variation of the Householder implementation of GMRES which may be useful in some circumstances on parallel computers. This variation is obtained by using simple formulas to express products of Householder transformations acting on vectors as operations involving matrix-vector products. In our experiments, this variation performed well while analogous variations of the Gram-Schmidt implementation, which use the classical Gram-Schmidt process, did not perform as effectively.

Notational conventions are as follows: Matrices are denoted by capital letters whereas vectors and scalars are denoted by lower case letters. Vector components and matrix entries are indicated by superscripts in parentheses, e. g., $v^{(i)}$ denotes the $i$ th component of the vector $v$. With or without subscripts or other distinguishing marks, the letters $H, J, L, P$, and $R$ always indicate matrices of the following respective types: upper-Hessenberg, Givens rotation, lower-triangular, Householder transformation, and upper-triangular. (See Golub and Van Loan [4] for definitions and properties.) The $i$ th canonical basis vector, i. e., the ith column of the identity matrix $I$, is denoted by $e_{i}$. Dimensions of vectors and matrices and, when appropriate, their (possibly) nonzero elements are implicit from the contexts in which they appear. For example, if $R$ is $p \times q$ upper-triangular and we write $R=\left(c e_{1}, H, h\right)$, 
then $H$ must be $p \times(q-2)$ upper-Hessenberg and $h$ must be a $p$-vector with zero components after the $q$ th.

2. The Householder implementation. We first formulate an algorithm which uses Householder transformations to generate orthonormal bases of Krylov subspaces. Similar use of Householder transformations is made in the references [3], [4] cited in the introduction.

Algorithm 2.1. Suppose $v_{1}$ is given with $\left\|v_{1}\right\|_{2}=1$.

1. Choose $P_{1}$ such that $P_{1} v_{1}=e_{1}$.

2. For $m=1,2, \ldots$, do:

a. Set $v_{m}=P_{1} \ldots P_{m} e_{m}$.

b. If $\left(v_{1}, A v_{1}, \ldots, A v_{m}\right)$ has rank $m$, then stop;

otherwise, choose $P_{m+1}$ such that

$P_{m+1} \ldots P_{1}\left(v_{1}, A v_{1}, \ldots, A v_{m}\right)$ is upper-triangular.

LEMMA 2.1. A set $\left\{v_{1}, \ldots, v_{m}\right\}$ generated by Algorithm 2.1 is an orthonormal basis of $K_{m}\left(v_{1}\right)$.

Proof. The lemma is trivially true if $m=1$, so suppose $m>1$. The facts that $P_{1} v_{1}=e_{1}$ and $P_{k} \ldots P_{1}\left(v_{1}, A v_{1}, \ldots, A v_{k-1}\right)$ is upper-triangular for $k=2, \ldots, m$ imply that for $k=2, \ldots, m$ the first $k-1$ components of the Householder vector determining $P_{k}$ are zero. It follows that for $1 \leq k \leq m, v_{j}=P_{1} \ldots P_{k} e_{j}$ for $j=1, \ldots, k$, i.e., that

$$
P_{1} \ldots P_{k}=\left(v_{1}, \ldots, v_{k}, \ldots\right), \quad 1 \leq k \leq m \text {. }
$$

In particular, $P_{1} \ldots P_{m}=\left(v_{1}, \ldots, v_{m}, \ldots\right)$, and so $\left\{v_{1}, \ldots, v_{m}\right\}$ is an orthonormal set.

It remains to show that $\mathcal{K}_{m}\left(v_{1}\right)=\operatorname{span}\left\{v_{1}, \ldots, v_{m}\right\}$. Certainly $K_{1}\left(v_{1}\right)=\operatorname{span}\left\{v_{1}\right\}$. Suppose that

$$
\mathcal{K}_{k}\left(v_{1}\right) \equiv \operatorname{span}\left\{v_{1}, A v_{1}, \ldots, A^{k-1} v_{1}\right\}=\operatorname{span}\left\{v_{1}, \ldots, v_{k}\right\}
$$

for some $k$ such that $1 \leq k<m$. Then

$$
\mathcal{K}_{k+1}\left(v_{1}\right) \equiv \operatorname{span}\left\{v_{1}, A v_{1}, \ldots, A^{k} v_{1}\right\}=\operatorname{span}\left\{v_{1}, A v_{1}, \ldots, A v_{k}\right\}
$$

Since $k<m,\left(v_{1}, A v_{1}, \ldots, A v_{k}\right)$ has rank $k+1$, and so $P_{k+1} \ldots P_{1}\left(v_{1}, A v_{1}, \ldots, A v_{k}\right)$ is an upper-triangular matrix which also has rank $k+1$. Consequently, the span of $\left\{v_{1}, A v_{1}, \ldots, A v_{k}\right\}$ is the span of the first $k+1$ columns of $P_{1} \ldots P_{k+1}$. It follows from (2.2) and (2.1) that $K_{k+1}\left(v_{1}\right)=\operatorname{span}\left\{v_{1}, \ldots, v_{k+1}\right\}$, and the induction is complete.

To use Algorithm 2.1 in implementing the GMRES method, we take $v_{1}= \pm r_{0} /\left\|r_{0}\right\|_{2}$, where for numerical soundness the sign is chosen so that the first component of $v_{1}$ is positive. At the $m$ th step of Algorithm 2.1, if $\left(v_{1}, A v_{1}, \ldots, A v_{m}\right)$ is of full rank, then we choose $P_{m+1}$ according to Algorithm 2.1 and set

$$
P_{m+1} \ldots P_{1}\left(A v_{1}, \ldots, A v_{m}\right)=H_{m}
$$

Writing $z=\left(v_{1}, \ldots, v_{m}\right) y$ for $y \in \mathbf{R}^{m}$, one has

$$
\begin{aligned}
\min _{z \in \mathcal{K}_{m}\left(r_{0}\right)}\left\|b-A\left(x_{0}+z\right)\right\|_{2} & =\min _{y \in \mathbf{R}^{m}}\left\|r_{0}-A\left(v_{1}, \ldots, v_{m}\right) y\right\|_{2} \\
& =\min _{y \in \mathbf{R}^{m}}\| \pm\| r_{0}\left\|_{2} e_{1}-H_{m} y\right\|_{2}
\end{aligned}
$$


It follows that $z_{m}=\left(v_{1}, \ldots, v_{m}\right) y_{m}$, where $y_{m}$ solves

$$
\min _{y \in \mathbf{R}^{m}}\| \pm\| r_{0}\left\|_{2} e_{1}-H_{m} y\right\|_{2}
$$

As in the GMRES implementation of [8], the least-squares problem (2.3) is solved by maintaining a factorization $H_{m}=Q_{m} R_{m}$, where $Q_{m}$ is a product of Givens rotations and $R_{m}$ is upper-triangular; these factors are updated with each increment of $m$. Then (2.3) is equivalent to

$$
\min _{y \in \mathbf{R}^{m}}\| \pm\| r_{0}\left\|_{2} Q_{m}^{T} e_{1}-R_{m} y\right\|_{2}
$$

and $y_{m}$ is found by solving an upper-triangular system. Note that the absolute value of the last coordinate of $\pm\left\|r_{0}\right\|_{2} Q_{m}^{T} e_{1}$ is $\left\|b-A x_{m}\right\|_{2}$, and as in the Gram-Schmidt implementation the residual can be found without having to compute the correction.

We give a complete outline of our Householder implementation of the GMRES method below. This implementation is of a "restarted" version of the method, in which the iteration proceeds for no more than a preset maximum number of times, which we assume to be less than or equal to $n$.

AlgORITHM 2.2. Suppose an initial approximate solution $x_{0}$, a tolerance $T O L$, and an iteration limit $M A X I T$ are given.

1. Compute $r_{0}=b-A x_{0}$, and determine $P_{1}$ such that $P_{1} r_{0}= \pm\left\|r_{0}\right\|_{2} e_{1} \equiv w$.

2. For $m=1,2, \ldots, M A X I T$, do:
a. Evaluate $v \equiv P_{m} \ldots P_{1} A P_{1} \ldots P_{m} e_{m}$.
b. If $v^{(m+1)}=\cdots=v^{(n)}=0$, then proceed to step (e); otherwise, continue.
c. Determine $P_{m+1}$ with a Householder vector having first $m$ components zero such that $P_{m+1} v$ has zero components after the $(m+1)$ st.
d. Overwrite $v \leftarrow P_{m+1} v$.
e. If $m>1$, overwrite $v \leftarrow J_{m-1} \ldots J_{1} v$.
f. If $v^{(m+1)}=0$, proceed to step (i); otherwise, continue.
g. Determine $J_{m}$ acting on components $m$ and $m+1$ such that $\left(J_{m} v\right)^{(m+1)}=0$.
h. Overwrite $v \leftarrow J_{m} v$ and $w \leftarrow J_{m} w$.
i. Set

$$
R_{m}= \begin{cases}(v), & \text { if } m=1 \\ \left(R_{m-1}, v\right), & \text { if } m>1\end{cases}
$$

j. If $\left|w^{(m+1)}\right| \leq T O L$ or $m=M A X I T$, then solve for $y_{m}$ and overwrite $x_{0}$ with $x_{m}$; otherwise, increment $m$.

3. Solve for $y_{m}$ and overwrite $x_{0}$ with $x_{m}$.
a. Determine $y_{m}$ which minimizes $\left\|w-R_{m} y\right\|_{2}$ by solving an $m \times m$ upper- triangular system with the first $m$ rows of $R_{m}$ as the coefficient matrix and the first $m$ components of $w$ as the right-hand side.

b. For $k=1, \ldots, m$, do:

Overwrite $x_{0} \leftarrow x_{0}+y_{m}^{(k)} P_{1} \ldots P_{k} e_{k}$.

c. If $\left|w^{(m+1)}\right| \leq T O L$, accept $x_{0}$ as the solution; otherwise, return to 1 .

In Algorithm 2.2, the Householder vectors determining $P_{1}, \ldots, P_{m}$ are stored instead of the orthonormal basis vectors $v_{1}, \ldots, v_{m}$. Each basis vector $v_{m}=P_{1} \ldots P_{m} e_{m}$ is generated when needed as part of larger computations in steps (2.a) and (3.b). Of 
course, the basis vectors generated in step (2.a) can be stored for later use in step (3.b) if this is preferable to regenerating them in step (3.b). The Householder vectors are of decreasing length, and they together with the upper-triangular matrices generated by the algorithm can be stored in an array of dimension $n \times(M A X I T+1)$. This is somewhat less storage than is required by the Gram-Schmidt implementation, which stores full-length orthonormal basis vectors as well as upper-triangular matrices. Since $M A X I T \ll n$ in most applications, this savings in storage is unlikely to be a major advantage.

Algorithm 2.2 requires more arithmetic than the Gram-Schmidt implementation because of the need to generate basis vectors in steps (2.a) and (3.b). This arithmetic is not necessary in the Gram-Schmidt implementation since orthonormal basis vectors are available from storage. Except for this, the arithmetic needed by the Householder and Gram-Schmidt implementations is about the same, and both require the same number of products of $A$ with vectors. For a more detailed comparison, we count the number of multiplications and products of $A$ with vectors required by the two algorithms, assuming that $M A X I T \ll n$ and ignoring terms less than $O(n)$.

In Algorithm 2.2, step (1) requires about $2 n$ multiplications and one $A$-product. For each $m$, steps (2.a) and (2.d) require about $4 \mathrm{~nm}$ multiplications and one $A$ product. If a total of $m$ iterations are performed in step (2), then about $2 n m(m+1)$ multiplications and $m A$-products are required. For each $k$, step (3.b) requires about $2 n(k-1)+n=2 n k-n$ multiplications, and so step (3.b) requires a total of about $n m^{2}$ multiplications. Thus Algorithm 2.2 requires about $n\left(3 m^{2}+2 m+2\right)$ multiplications and $m+1 A$-products to carry out $m$ GMRES iterations and to compute the resulting approximate solution. The Gram-Schmidt implementation requires one $A$-product to compute the initial residual and about $2 n$ multiplications to normalize it. At the $m$ th GMRES iteration, one $A$-product and about $2 n(m+1)$ multiplications are needed to produce the next orthonormal basis vector. If a total of $m$ iterations are performed, then about $n m(m+3)$ multiplications and $m A$-products are required. Computing the approximate solution after $m$ iterations requires about $n m$ additional multiplications. The Gram-Schmidt implementation then requires about $n\left(m^{2}+4 m+2\right)$ multiplications and $m+1 A$-products to carry out $m$ GMRES iterations and compute the resulting approximate solution. There is a variation of the Gram-Schmidt implementation in which successive basis vectors are "reorthogonalized", i. e., subjected again to the Gram-Schmidt process, in the hope of reducing loss of orthogonality due to numerical error. If reorthogonalization is carried out before normalization at every GMRES iteration, then this variation requires about $n\left(2 m^{2}+5 m+2\right)$ multiplications and $m+1$ $A$-products to carry out $m$ GMRES iterations and compute the resulting approximate solution.

The cost of the additional arithmetic required by Algorithm 2.2 may be significant; however, it may not be prohibitive if $M A X I T$ is small or if computation is done on vector computers which effectively reduce the expense of applying Householder transformations to vectors. Also, it may not be a very significant part of the overall computational expense in applications in which $A$-products are relatively costly or in which the GMRES iterations themselves are only a small part of a larger computation. One such application is that given by Brown and Hindmarsh [2] to stiff ODE solving. There, $A$-products are approximated by forming difference quotients of values of nonlinear functions and the iterative solution of linear systems is itself only a part of the total effort of solving stiff ODE initial-value problems. Finally, our numerical experiments suggest that the variation of the Householder implementation given in the next section may offer an opportunity for effectively exploiting parallelism in 
some instances. Analogous variations of the Gram-Schmidt implementation use the classical Gram-Schmidt process and are not as numerically sound.

A numerical experiment. We compared the performance of the Householder and Gram-Schmidt implementations of the GMRES method on a nonsymmetric linear system arising from the discretization of the boundary value problem

$$
\begin{aligned}
\Delta u+c u+d \frac{\partial u}{\partial x} & =f & & \text { in } D \\
u & =0 & & \text { on } \partial D
\end{aligned}
$$

where $D=[0,1] \times[0,1]$ and $c \geq 0$ and $d$ are constants. In our trials, we took $f(x) \equiv 1$ and used a $100 \times 100$ mesh of discretization points on $D$. We used a variety of values of $c$ and $d$ ranging from 1 to 1000, and the maximum number of GMRES iterations allowed before restarting (MAXIT in Algorithm 2.2) ranged from 5 to 50. No preconditioning was used in order to keep the issues of interest clear. Computing was done in single precision on a Digital Equipment Corporation MicroVAX II running Ultrix and using the f77 Fortran compiler.

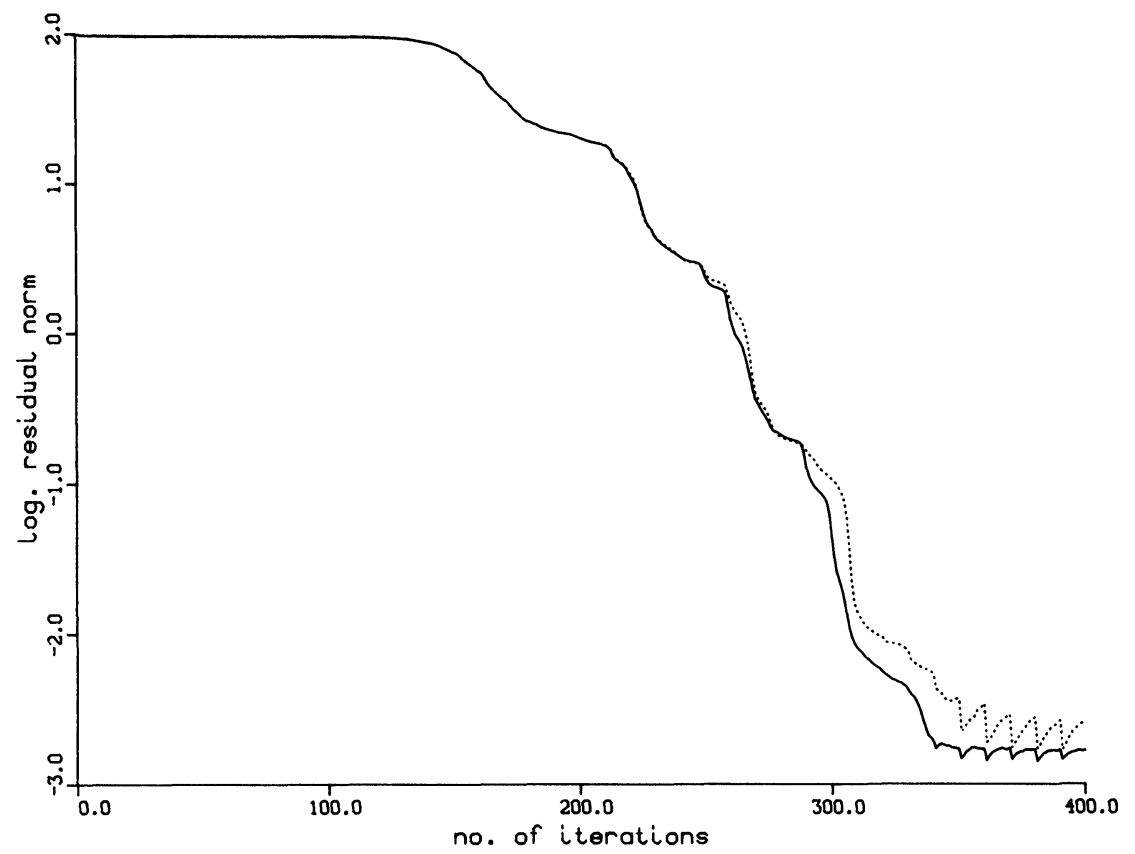

FIG. 1. The solid line is from the Householder implementation. The dotted line is from the Gram-Schmidt implementation.

For each trial, we plotted the logarithm of the residual norm versus the number of iterations for the two implementations. It is important to note that in these trials the residual norms were determined "from scratch," i.e., at each iteration, the current approximate solution was computed and multiplied by the coefficient matrix, then the right-hand side vector was subtracted and the norm taken. In particular, the residual norms were not taken to be the values maintained by the GMRES implementations. An important aspect of these trials is that they show the unreliability of these values as the limits of residual reduction are neared.

The results shown in Figure 1 are typical. They were obtained with $c=d=100$ and with the maximum number of iterations allowed before restarting equal to 10 . Note 
the "sawtooth" pattern of residual-norm levels which appears as the limits of residual reduction are reached. This pattern continues unabated as additional iterations are made. It is exhibited by both implementations, but it is much more pronounced for the Gram-Schmidt implementation. We also tested the Gram-Schmidt implementation with reorthogonalization in this case and found the results to be visually indistinguishable from those produced by the Gram-Schmidt implementation without reorthogonalization.

3. A variation. We now give a variation of Algorithm 2.2 in which the products of Householder transformations acting on vectors are replaced by operations which to a large extent can be performed concurrently. Our hope is that this variation will provide an effective way of exploiting parallelism in circumstances which favor evaluating matrix-vector products in parallel. We count as matrix-vector products such operations as computing the inner products of a vector with the vectors in some set and accumulating a sum of scalar multiples of vectors in some set. The modified Gram-Schmidt process is inherently sequential, and the only apparent variations of the Gram-Schmidt implementation of GMRES which can take advantage of parallelism similarly use the potentially unstable classical Gram-Schmidt process. We have no proof of the numerical superiority of the variation of the Householder implementation given here, but we describe a numerical experiment below in which it performs significantly better than the Gram-Schmidt implementation using the classical GramSchmidt process, even when reorthogonalization is done.

The variation of Algorithm 2.2 is based on the following: If $v, w_{1}, z_{1}, \ldots, w_{m}, z_{m}$ are vectors, then one can write

$$
\left(I+w_{m} z_{m}^{T}\right) \ldots\left(I+w_{1} z_{1}^{T}\right) v=v+d^{(1)} w_{1}+\cdots+d^{(m)} w_{m}
$$

where $d^{(1)}, \ldots, d^{(m)}$ are determined by

$$
\begin{aligned}
& d^{(1)}=z_{1}^{T} v, \\
& d^{(k)}=z_{k}^{T} v+d^{(1)} z_{k}^{T} w_{1}+\cdots+d^{(k-1)} z_{k}^{T} w_{k-1}, \quad k=2, \ldots, m .
\end{aligned}
$$

Note that (3.1) is equivalent to specifying $d=\left(d^{(1)}, \ldots, d^{(m)}\right)^{T}$ by solving $L d=$ $\left(z_{1}, \ldots, z_{m}\right)^{T} v$ using forward substitution, where

$$
L=\left(\begin{array}{cccc}
1 & 0 & \cdots & 0 \\
-z_{2}^{T} w_{1} & 1 & \cdots & 0 \\
\vdots & \ddots & \ddots & \vdots \\
-z_{m}^{T} w_{1} & \cdots & -z_{m}^{T} w_{m-1} & 1
\end{array}\right)
$$

Then one can write

$$
\left(I+w_{m} z_{m}^{T}\right) \ldots\left(I+w_{1} z_{1}^{T}\right) v=\left\{I+\left(w_{1}, \ldots, w_{m}\right) L^{-1}\left(z_{1}, \ldots, z_{m}\right)^{T}\right\} v
$$

with $\mathrm{L}$ given by (3.2). It follows in particular that if one has Householder transformations $P_{k}=I-2 u_{k} u_{k}^{T}, k=1, \ldots, m$, and a vector $v$, then

$$
P_{m} \ldots P_{1} v=\left\{I-2 U_{m} L_{m}^{-1} U_{m}^{T}\right\} v
$$

where

$$
U_{m}=\left(u_{1}, \ldots, u_{m}\right) \quad \text { and } \quad L_{m}=\left(\begin{array}{cccc}
1 & 0 & \ldots & 0 \\
2 u_{2}^{T} u_{1} & 1 & \ldots & 0 \\
\vdots & \ddots & \ddots & \vdots \\
2 u_{m}^{T} u_{1} & \ldots & 2 u_{m}^{T} u_{m-1} & 1
\end{array}\right)
$$


Similarly,

$$
P_{1} \ldots P_{m} v=\left\{I-2 U_{m}\left(L_{m}^{T}\right)^{-1} U_{m}^{T}\right\} v
$$

where $U_{m}$ and $L_{m}$ are given by (3.4). It is understood that the steps in evaluating $P_{m} \ldots P_{1} v$ using the right-hand side of (3.3) are the following:

1. Evaluate $U_{m}^{T} v$.

2. Solve $L_{m} d=-2 U_{m}^{T} v$ by forward substitution.

3. Evaluate

$$
v+U_{m} d=\left(v, U_{m}\right)\left(\begin{array}{l}
1 \\
d
\end{array}\right) .
$$

Similar remarks hold for evaluating the right-hand side of (3.5).

The full outline of the variation of Algorithm 2.2 is given in the following algorithm.

ALGORITHM 3.1. Suppose an initial approximate solution $x_{0}$, a tolerance $T O L$, and an iteration limit $M A X I T$ are given.

1. Compute $r_{0}=b-A x_{0}$, determine $P_{1}=I-2 u_{1} u_{1}^{T}$ such that $P_{1} r_{0}= \pm\left\|r_{0}\right\|_{2} e_{1} \equiv w$, and set $U_{1}=\left(u_{1}\right)$ and $L_{1}=(1)$.

2. For $m=1,2, \ldots, M A X I T$, do:
a. Evaluate $v \equiv\left\{I-2 U_{m} L_{m}^{-1} U_{m}^{T}\right\} A\left\{I-2 U_{m}\left(L_{m}^{T}\right)^{-1} U_{m}^{T}\right\} e_{m}$.
b. If $v^{(m+1)}=\cdots=v^{(n)}=0$, then proceed to step (e); otherwise, continue.
c. Determine $P_{m+1}=I-2 u_{m+1} u_{m+1}^{T}$ with $u_{m+1}$ having first $m$ compo- nents zero such that $P_{m+1} v$ has zero components after the $(m+1) s t$.
d. Overwrite $v \leftarrow P_{m+1} v$.
e. If $m>1$, overwrite $v \leftarrow J_{m-1} \ldots J_{1} v$.
f. If $v^{(m+1)}=0$, proceed to step (i); otherwise, continue.
g. Determine $J_{m}$ acting on components $m$ and $m+1$ such that $\left(J_{m} v\right)^{(m+1)}=0$.
h. Overwrite $v \leftarrow J_{m} v$ and $w \leftarrow J_{m} w$.
i. Set

$$
R_{m}= \begin{cases}(v), & \text { if } m=1 \\ \left(R_{m-1}, v\right), & \text { if } m>1\end{cases}
$$

j. If $\left|w^{(m+1)}\right| \leq T O L$ or $m=M A X I T$, then solve for $y_{m}$ and overwrite $x_{0}$ with $x_{m}$; otherwise, continue.

k. Set $U_{m+1}=\left(U_{m}, u_{m+1}\right)$, evaluate

$$
L_{m+1}=\left(\begin{array}{cc}
L_{m} & 0 \\
2 u_{m+1}^{T} U_{m} & 1
\end{array}\right)
$$

and increment $m$.

3. Solve for $y_{m}$ and overwrite $x_{0}$ with $x_{m}$.

a. Determine $y_{m}$ which minimizes $\left\|w-R_{m} y\right\|_{2}$ by solving an $m \times m$ uppertriangular system with the first $m$ rows of $R_{m}$ as the coefficient matrix and the first $m$ components of $w$ as the right-hand side.

b. Overwrite $x_{0} \leftarrow x_{0}+\left\{I-2 U_{m}\left(L_{m}^{T}\right)^{-1} U_{m}^{T}\right\}\left(e_{1}, \ldots, e_{m}\right) y_{m}$.

c. If $\left|w^{(m+1)}\right| \leq T O L$, accept $x_{0}$ as the solution; otherwise, return to 1 .

A numerical experiment. We compared the performance of Algorithm 3.1 with that of the Gram-Schmidt implementation of GMRES using the classical Gram-Schmidt process with and without reorthogonalization. There was essentially no difference in 
the performance of these implementations on the problem considered in the preceding section. However, the difference was significant on a contrived problem (1.1) in which

$$
A=\left(\begin{array}{ccccc}
1 & 0 & \ldots & 0 & \alpha \\
0 & 2 & \ldots & 0 & 0 \\
\vdots & \vdots & & \vdots & \vdots \\
0 & 0 & \ldots & 0 & n
\end{array}\right) \quad \text { and } \quad b=\left(\begin{array}{c}
1 \\
1 \\
\vdots \\
1
\end{array}\right)
$$

As in the numerical experiment in the preceding section, we plotted the logarithm of the residual norm versus the number of iterations for these implementations, with the residual norm computed "from scratch." The computing environment and precision were the same as before, and again no preconditioning was used.

Algorithm 3.1 performed markedly better than the Gram-Schmidt implementations in our trials. The results shown in Figure 2 are typical. These results were obtained with $n=100, \alpha=2,000$, and with the maximum number of iterations allowed before restarting equal to 32 . The Gram-Schmidt implementation without reorthogonalization performed very poorly. The Gram-Schmidt implementation with reorthogonalization performed considerably better but did not achieve the final residual reduction of Algorithm 3.1 and appeared to suffer greater instability as the limits of residual reduction were approached. Although not shown, the performance of Algorithm 2.2 was about the same as that of Algorithm 3.1 in this and other trials.

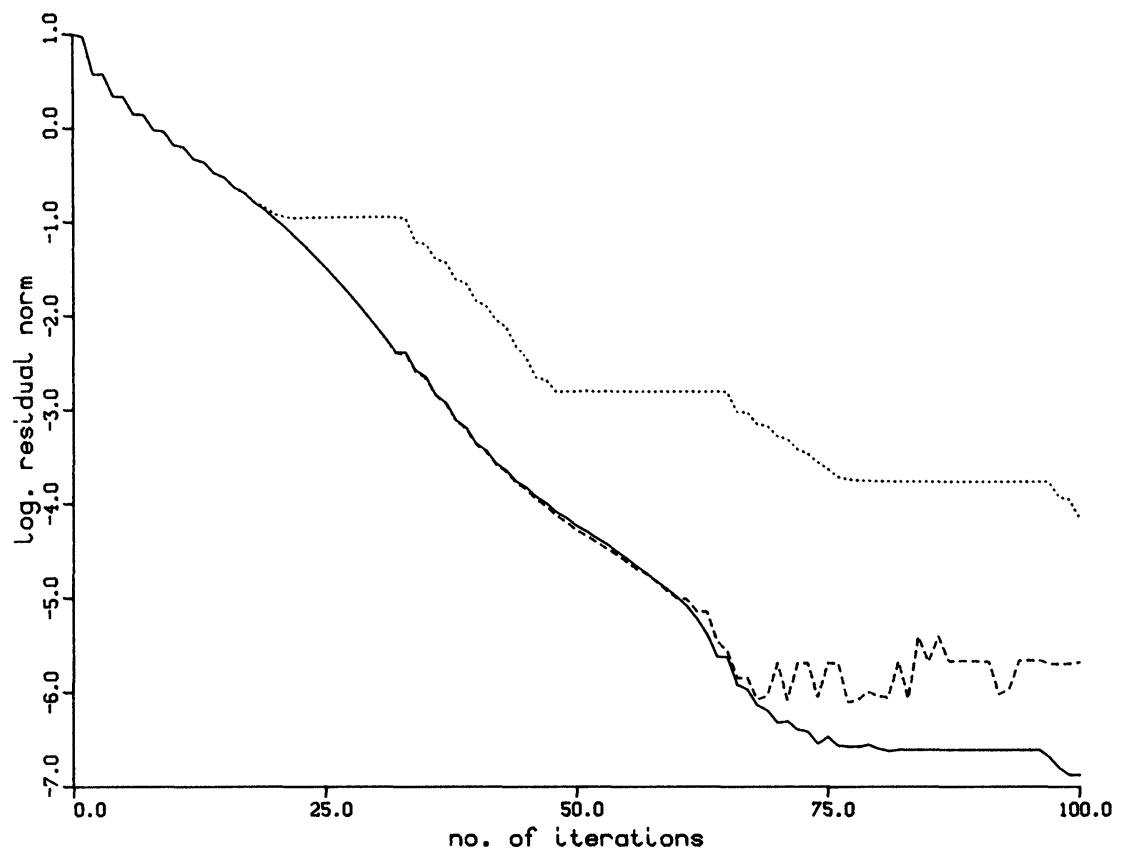

FIG. 2. The solid line is from the variation of the Householder implementation. The dashed and dotted lines are from the Gram-Schmidt implementations using the classical Gram-Schmidt process with and without reorthogonalization, respectively.

Acknowledgment. The author thanks Youcef Saad for providing the two test problems and the Gram-Schmidt implementation of the GMRES method used in the numerical experiments. 


\section{REFERENCES}

[1] A. BJORCK, Solving linear least-squares problems by Gram-Schmidt orthogonalization, BIT, 7 (1967), pp. 1-21.

[2] P. N. BROWN AND A. C. HINDMARSH, Matrix-free methods for stiff systems of ODE's, SIAM J. Numer. Anal., 23 (1986), pp. 610-638.

[3] G. H. GOLUB, R. UNDERWOOD AND J. H. Wilkinson, The Lanczos algorithm for the symmet ric $A x=\lambda B x$ problem, Computer Science Dept. Report STAN-CS-72-270, Stanford Univ., Stanford, CA, 1972.

[4] G. H. Golub AND C. F. VAN LOAN, Matrix Computations, Johns Hopkins University Press, Baltimore, MD, 1983.

[5] A. C. HiNdMARSh AND H. F. WALKER, A note on a Householder transformation implementation of the GMRES method, Lawrence Livermore Nat. Lab. Tech. Report UCID-20899, 1986.

[6] Y. SAAD, Krylov subspace methods for solving large unsymmetric linear systems, Math. Comp., 37 (1981), pp. 105-126.

[7] - Practical use of some Krylov subspace methods for solving indefinite and nonsymmetric linear systems, this Journal, 5 (1984), pp. 203-228.

[8] Y. SAAD AND M. H. SCHULTZ, GMRES: a generalized minimal residual algorithm for solving nonsymmetric linear systems, this Journal, 7 (1986), pp. 856-869.

[9] H. F. WALKER, Implementation of the GMRES and Arnoldi methods using Householder transformations, Lawrence Livermore Nat. Lab. Tech. Report UCRL-93589, 1985.

[10] L. B. WIGTON, N. J. YU, AND D. P YOUNG, GMRES acceleration of fluid dynamics codes, Proceedings of the American Institute of Aeronautics and Astronautics 7th Computational Fluid Dynamics Conference, Cincinnati, OH, July 15-17, 1985, pp. 67-74. 